\title{
Mesoscopic effects in the fractional quantum Hall regime
}

\author{
Michael R. Geller ${ }^{a}$ and Daniel Loss ${ }^{b}$ \\ ${ }^{a}$ Department of Physics and Astronomy, University of Georgia, Athens, Georgia 30602-2451 \\ ${ }^{b}$ Department of Physics and Astronomy, University of Basel, Klingelbergstrasse 82, 4056 Basel, \\ Switzerland
}

(Version: October 26, 2017)

\begin{abstract}
Edge states of the quantum Hall fluid provide an opportunity to study mesoscopic effects in a highly correlated electron system that is both experimentally accessible and theoretically tractable. In this paper we review recent work on the persistent current and Aharonov-Bohm magnetoconductance oscillations in the fractional quantum Hall effect regime.
\end{abstract}

Keywords: chiral Luttinger liquid, Aharonov-Bohm effect, fractional quantum Hall effect

Corresponding author:

Michael R. Geller

Department of Physics

University of Georgia

Athens, GA 30602

Fax: 706-542-2492

Email: mgeller@hal.physast.uga.edu 


\section{INTRODUCTION}

In pioneering work, Wen [1] used the Chern-Simons theory of the bulk fractional quantum Hall effect (FQHE) to show that the edge states there should be chiral Luttinger liquids (CLL). As in the nonchiral Luttinger liquid, electron-electron interactions in the CLL play an essential role and lead to physical properties of the FQHE edge states that can be dramatically different than that in the integral quantum Hall effect regime.

In this paper we present results of an ongoing study of mesoscopic effects in the CLL. After a review of the bosonization of finite-size FQHE edge states, we discuss chiral persistent currents that are predicted to have a universal non-Fermi-liquid temperature dependence, and the Aharonov-Bohm (AB) magnetoconductance oscillations in the CLL.

\section{THE FINITE-SIZE CHIRAL LUTTINGER LIQUID}

We begin with a brief review of the bosonization and momentum-space quantization of the finite-size CLL [2 4]. The dynamics of edge states in the FQHE regime is governed by the Euclidian action [5]

$$
S_{ \pm}=\frac{1}{4 \pi g} \int_{0}^{L} d x \int_{0}^{\beta} d \tau \partial_{x} \phi_{ \pm}\left( \pm i \partial_{\tau} \phi_{ \pm}+v \partial_{x} \phi_{ \pm}\right)
$$

where $\rho_{ \pm}= \pm \partial_{x} \phi_{ \pm} / 2 \pi$ is the charge density fluctuation for right $(+)$ or left (-) moving electrons, $g=1 / q$ (with $q$ odd) is the bulk filling factor, and $v$ is the edge-magnetoplasmon

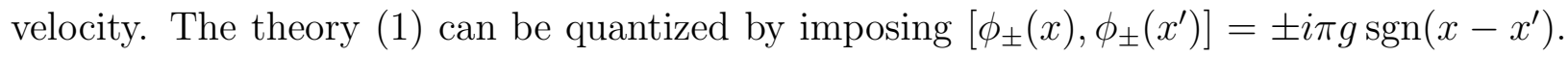
We then decompose $\phi_{ \pm}$into a nonzero-mode contribution $\phi_{ \pm}^{\mathrm{p}}$ satisfying periodic boundary

conditions and a zero-mode part $\phi_{ \pm}^{0}$. The nonzero-mode part may be expanded in a basis of Bose annihilation and creation operators as

$$
\phi_{ \pm}^{\mathrm{p}}(x)=\sum_{k \neq 0} \theta( \pm k) \sqrt{\frac{2 \pi g}{|k| L}}\left(a_{k} e^{i k x}+a_{k}^{\dagger} e^{-i k x}\right),
$$

and the zero-mode field may be written as

$$
\phi_{ \pm}^{0}(x)= \pm \frac{2 \pi}{L} N_{ \pm} x-g \chi_{ \pm}
$$


where $\chi_{ \pm}$is an Hermitian phase operator conjugate to $N_{ \pm}$satisfying $\left[\chi_{ \pm}, N_{ \pm}\right]=i$. Here $N_{ \pm} \equiv \int_{0}^{L} d x \rho_{ \pm}$is the charge of an excited state relative to the ground state. Eqns. (2) and (3) may be used to write the normal-ordered CLL Hamiltonian as

$$
H_{ \pm}=\frac{\pi v}{g L} N_{ \pm}^{2}+\sum_{k} \theta( \pm k) v|k| a_{k}^{\dagger} a_{k}
$$

The electron field operators can be bosonized as

$$
\psi_{ \pm}(x)=\frac{1}{\sqrt{2 \pi a}} e^{i \phi_{ \pm}(x) / g} e^{ \pm i \pi x / g L}
$$

where $a$ is a microscopic cutoff length. The phase factor $e^{ \pm i \pi x / g L}$, which has the effect of disentangeling the charge and phase operators in the zero-mode, is necessary for bosonizaton in a finite-size system; see Ref. [4] for further discussion. (Similar factors are known to occur in the bosonization of nonchiral finite-size systems [6.74.) Imposing periodic boundary conditions on the electron field operators leads to the requirement that the allowed eigenvalues of $N_{ \pm}$are given by 41

$$
N_{ \pm}=n g
$$

where $n$ is any integer, which means that there exists fractionally charged excitations, as expected in a FQHE system.

\section{PERSISTENT CURRENT IN THE CHIRAL LUTTINGER LIQUID}

In a macroscopic FQHE edge state, an equilibrium edge current exists even in the absence of an AB flux or twisted boundary conditions. The magnitude of this current is universal and in the absence of disorder is given by [8] $I_{\text {edge }}=g e \omega_{\mathrm{c}} / 4 \pi+e \tilde{\epsilon}_{\mathrm{qh}} / 2 \pi$, where $\omega_{\mathrm{c}}$ is the cyclotron frequency and $\tilde{\epsilon}_{\mathrm{qh}}$ is the proper quasihole energy [9] of the Laughlin state at filling factor $g=1 / q$.

We now couple the edge state to an $\mathrm{AB}$ flux $\Phi$. The grand-canonical partition function of the mesoscopic edge state factorizes into a zero-mode contribution, 


$$
Z^{0}=\sum_{n=-\infty}^{\infty} e^{-g \pi^{2}\left(T_{0} / T\right)(n-\varphi)^{2}}
$$

which depends on $\Phi$, and a flux-independent contribution $Z^{\mathrm{p}}$ from the nonzero-modes. Here $T_{0} \equiv v / \pi L$ and $\varphi \equiv \Phi / \Phi_{0}$, with $\Phi_{0} \equiv h c / e$ the flux quantum. Note that if $N_{ \pm}$were restricted to be an integer then the period of these equilibrium $\mathrm{AB}$ oscillations would be $\Phi_{0} / g$. The allowed fractionally charged excitations (6) are therefore responsible for restoring the $\mathrm{AB}$ period to $\Phi_{0}$, as is well-known in other contexts [10].

The edge current induced from the additional flux $\Phi$ is found to be [11]

$$
I \equiv-\frac{\partial \Omega}{\partial \Phi}=\frac{2 \pi T}{\Phi_{0}} \sum_{n=1}^{\infty}(-1)^{n} \frac{\sin (2 \pi n \varphi)}{\sinh \left(n q T / T_{0}\right)},
$$

where $\Omega$ is the grand-canonical potential. At zero temperature, this chiral persistent current has an amplitude $g \frac{e v}{L}$. Note that $I$ is renormalized by interactions in precisely the same

way as in a nonchiral Luttinger liquid [6]. For $T \gg T_{0}$ the amplitude decays as $g \frac{e v}{L} e^{-q T / T_{0}}$. Because these persistent currents are chiral, there is no backscattering from impurities and hence no amplitude reduction from weak disorder. The temperature dependence of the orbital magnetic response of a FQHE edge state may therefore be another ideal system to observe non-Fermi-liquid mesoscopic behavior.

We note here that the persistent current in a FQHE annulus (the sum of currents from the inner and outer edge states) has been studied recently by Kettemann [12].

\section{AHARONOV-BOHM EFFECT IN THE CHIRAL LUTINGER LIQUID}

The first experimental observation of a CLL was made by Milliken, Umbach, and Webb [13], who measured the tunneling current between two filling factor $1 / 3$ edge states in a quantum-point-contact geometry. As the gate voltage was varied, resonance peaks in the conductance were observed to have the correct CLL temperature dependence as predicted by Moon and coworkers [14], following earlier related work by Kane and Fisher [15]. However, recent experiments by Franklin et al. [16] and by Maasilta and Goldman [17 on tunneling 
between FQHE edge states through an additional edge state circling an antidot have reported Fermi liquid behavior.

We have studied this problem using CLL theory and found that the transport properties of the quantum-point-contact system and the antidot system differ in two important ways: The first is that mesoscopic effects are very important in the latter. When the thermal length $L_{\mathrm{T}} \equiv v / T$ becomes smaller than the circumference $L$ of the antidot edge state, the AB oscillations become washed out, and, at the same time, acquire a temperature dependence that is similar to a chiral Fermi liquid. An experiment performed at a temperature significantly above the point of crossover, $T_{0} \equiv v / \pi L$, is therefore expected to observe nearly Fermi liquid behavior for many mesoscopic quantities. The second difference is that in contrast with the quantum-point-contact geometry, where it is reasonable to assume that there exist conditions of destructive interference that lead to perfect resonances, the resonances in the antidot geometry, which are controlled by the AB effect, are never perfect, even at zero temperature.

A scaling analysis of the AB effect in the CLL has been given in Ref. [4], following similar work by Moon et al. [14]. The scaling behavior in the antidot system differs from that in the point contact. Although the renormalization group $(\mathrm{RG})$ equations are the same, their physical implications are different. As mentioned above, the AB effect in a chiral system does not lead to destructive interference, so it is not possible to tune the bare quasiparticle tunneling amplitude all the way to zero. This means that at low enough temperatures the system will always be in the strong-antidot-coupling regime, for all values of the AB flux and gate voltages. The sharp non-Fermi-liquid resonance studied in the quantum-point-contact geometry, having a width varying with temperature as $T^{1-g}$, is not expected in the antidot geometry at the lowest temperatures. Furthermore, the scale $T_{0}$ plays an important role. Whereas in the quantum-point-contact geometry the universal RG trajectory implies a oneparameter universal scaling function $\tilde{G}(X)$, where $X$ depends on the temperature, the RG trajectory in the antidot problem, which we also predict to be universal for sufficiently low $T_{0}$, leads instead to a two-parameter universal scaling function $\tilde{G}(X, Y)$, where $Y$ depends 
on the size of the antidot and is temperature independent. The scaling function $\tilde{G}(X, Y)$ contains all the mesoscopic effects associated with the finite-size edge state. In the $Y \rightarrow 0$ limit $\tilde{G}(X, Y)$ reduces to the one-parameter function $\tilde{G}(X)$ calculated by Moon et al. [14] and by Fendley, Ludwig, and Saleur [18].

The strong-antidot-coupling regime of the $\mathrm{AB}$ effect can be studied with perturbation theory [19,20]. Here we shall allow both electron and quasiparticle tunneling, the latter being important for the douple quantum-point-contact system studied by Chamon and coworkers [20]. The Hamiltonian is $H=H_{0}+\delta H$, where $H_{0}=H_{\mathrm{L}}+H_{\mathrm{R}}$ is a sum of Hamiltonians of the form (4) and

$$
\delta H=v \sum_{m} \sum_{i} \Gamma_{m i} B_{m i}+\text { h.c. },
$$

where $B_{m i} \equiv e^{i m \phi_{\mathrm{L}}\left(x_{i}\right)} e^{-i m \phi_{\mathrm{R}}\left(x_{i}\right)} / 2 \pi a$ is an $m$-quasiparticle tunneling operator (for macroscopic edge states) acting at point $x_{i}$. The current $I \equiv\left\langle\dot{N}_{\mathrm{R}}\right\rangle$ to first order in $\delta H$ is found to be

$$
I=\left.\sum_{m} 2 m g \operatorname{Im} \sum_{i j} \Gamma_{m i} \Gamma_{m j}^{*} X_{i j}^{m}(\omega)\right|_{\omega=m g V},
$$

where $X_{i j}^{m}(\omega)$ is the Fourier transform of $X_{i j}^{m}(t) \equiv-i \Theta(t)\left\langle\left[B_{m i}(t), B_{m j}^{\dagger}(0)\right]\right\rangle$. Each term in (10) corresponds to a process occurring with a probability proportional to $\left|\Gamma_{i} \Gamma_{j}\right|$. The local terms $X_{11}$ and $X_{22}$ describe independent tunneling at $x_{1}$ and $x_{2}$, whereas the nonlocal terms $X_{12}$ and $X_{21}$ describe coherent tunneling; the AB phase naturally couples only to the latter. The zero-temperature voltage dependence of (10) follows immediately from the local scaling dimension $\Delta=m^{2} g / 2$ of $e^{i m \phi_{ \pm}}$, which implies that $X_{i j}^{m}(t) \sim(1 / t)^{2 m^{2} g}$ and hence $X_{i j}^{m}(\omega) \sim \omega^{2 m^{2} g-1}$. Therefore, at zero temperature, $I \sim V^{2 m^{2} g-1}$ for low voltages, which is the same power-law behavior as predicted by the RG equations [21].

Restricting ourselves now to electron $(m=q)$ tunneling, the current can be written as $I=I_{0}+I_{\mathrm{AB}} \cos \left[2 \pi\left(\frac{\mu}{\Delta \epsilon}+\varphi\right)\right]$. The exact IV relation for the $g=1 / 3$ CLL is given in Ref. 19. Here we summarize our results for general $q$ as a function of temperature for fixed voltage, first for $V \ll T_{0}$ and then for $V \gg T_{0}$. 


\section{A. Low voltage regime: $V \ll T_{0}$}

There are three temperature regimes here. When $T \ll V \ll T_{0}$, both $I_{0}$ and $I_{\mathrm{AB}}$ are temperature independent but have nonlinear behavior, varying with voltage as

$$
I \propto V^{2 q-1}
$$

When the temperature exceeds $V$, the response becomes linear. When $V \ll T \ll T_{0}$, both $G_{0}$ and $G_{\mathrm{AB}}$ vary with temperature as

$$
G \propto\left(\frac{T}{T_{F}}\right)^{2 q-2}
$$

where $T_{\mathrm{F}} \equiv v / a$ is an effective Fermi temperature.

At a temperature near $T_{0}$, we find that $G_{\mathrm{AB}}$ for the CLL displays a pronounced maximum.

Increasing the temperature further we cross over into the $V \ll T_{0} \ll T$ regime where $G_{0}$ scales as in (12), but

$$
G_{\mathrm{AB}} \propto\left(\frac{T}{T_{0}}\right)\left(\frac{T}{T_{\mathrm{F}}}\right)^{2 q-2} e^{-q T / T_{0}} .
$$

Thus $G_{\mathrm{AB}}$ exhibits a crossover from the well-known $T^{2 q-2}$ Luttinger liquid behavior to a new scaling behavior which is much closer to a chiral Fermi liquid $(q=1)$. Careful measurements in this experimentally accessible regime should be able to distinguish between a Fermi liquid and this predicted nearly Fermi-liquid temperature dependence.

\section{B. High voltage regime: $V \gg T_{0}$}

Again there are three temperature regimes. For the lowest temperatures $T \ll T_{0} \ll V$, the response is again temperature independent and nonlinear. The direct term varies with voltage according to

$$
I_{0} \propto V^{2 q-1}
$$

as in the lowest temperature, low voltage regime. However, the flux-dependent part of the current is now much more interesting, involving power-laws times Bessel functions of the 
ratio $V / 2 \pi T_{0}=\pi V / \Delta \epsilon$. As the temperature is increased further to $T_{0} \ll T \ll V$, we find a crossover to an interesting high-temperature nonlinear regime. Here $I_{0} \propto V^{2 q-1}$ as before, but now

$$
I_{\mathrm{AB}} \propto\left(\frac{T}{T_{0}}\right)^{q} e^{-q T / T_{0}} V^{q-1} \sin \left(\frac{V}{2 \pi T_{0}}\right)
$$

Therefore, the nonlinear response at fixed temperature can also be used to distinguish between Fermi liquid and Luttinger liquid behavior, even at relatively high temperatures.

When the temperature exceeds $\mathrm{V}$, the response finally becomes linear. When $T_{0} \ll V \ll$ $T, G_{0}$ scales as in (12) whereas $G_{\mathrm{AB}}$ scales as in (13).

The strong-antidot-coupling regime studied here is ideal for experimental investigation because the exact current-voltage relation is known, and the low-temperature crossover from weak to strong coupling [4] does not complicate the analysis. If, by an appropriate choice of gate voltages, the antidot system starts in the strongly coupled regime, then it will stay in this regime throughout the relevant ranges of temperature and magnetic field.

This work has been supported by the NSERC of Canada. 


\section{REFERENCES}

[1] X. G. Wen, Phys. Rev. Lett. 64, 2206 (1990); Phys. Rev. B 43, 11025 (1991).

[2] F. D. M. Haldane, J. Phys. C 14, 2585 (1981).

[3] X. G. Wen, Phys. Rev. B 41, 12838 (1990).

[4] M. R. Geller and D. Loss, Phys. Rev. B (in press).

[5] X. G. Wen, Int. J. Mod. Phys. B 6, 1711 (1992).

[6] D. Loss, Phys. Rev. Lett. 69, 343 (1992).

[7] D. L. Maslov, M. Stone, P. M. Goldbart, and D. Loss, Phys. Rev. B 53, 1548 (1996).

[8] M. R. Geller and G. Vignale, Phys. Rev. B 52, 14137 (1995).

[9] B. I. Halperin, Phys. Rev. Lett. 52, 1583 (1984); R. Morf and B. I. Halperin, Phys. Rev. B 33, 2221 (1986).

[10] P. A. Lee, Phys. Rev. Lett. 65, 2206 (1990); S. A. Kivelson, Phys. Rev. Lett. 65, 3369 $(1990)$

D. J. Thouless and Y. Gefen, Phys. Rev. Lett. 66, 806 (1991).

[11] M. R. Geller, D. Loss, and G. Kirczenow, Superlattices and Microstructures 21, 49 (1997).

[12] S. Kettemann, Phys. Rev. B 55, 2512 (1997).

[13] F. P. Milliken, C. P. Umbach, and R. A. Webb, Solid State Commun. 97, 309 (1996).

[14] K. Moon, H. Yi, C. L. Kane, S. M. Girvin, and M. P. A. Fisher, Phys. Rev. Lett. 71, 4381 (1993).

[15] C. L. Kane and M. P. A. Fisher, Phys. Rev. Lett. 68, 1220 (1992).

[16] J. D. F. Franklin et al., Surf. Sci. 361, 17 (1996). 
[17] I. J. Maasilta and V. J. Goldman, Phys. Rev. B 55, 4081 (1997).

[18] P. Fendley, A. W. W. Ludwig, and H. Saleur, Phys. Rev. Lett. 74, 3005 (1995).

[19] M. R. Geller, D. Loss, and G. Kirczenow, Phys. Rev. Lett. 77, 5110 (1996).

[20] C. de C. Chamon, D. E. Freed, S. A. Kivelson, S. L. Sondhi, and X. G. Wen, Phys. Rev. B 55, 2331 (1997).

[21] Here one solves the RG flow equations of Ref. [14], finding that the renormalized $m$ quasiparticle tunneling rate at an energy scale $\epsilon$ is $\Gamma_{m}^{\prime}=\left(\epsilon / \epsilon_{\mathrm{F}}\right)^{m^{2} g-1} \Gamma_{m}$, where $\epsilon_{\mathrm{F}} \equiv v / a$ is the effective Fermi energy and $\Gamma_{m}$ is the bare value. Cutting off the scaling at $V$ leads to $I=\left|\Gamma_{m}^{\prime}\right|^{2} \frac{e^{2}}{h} \cdot V \sim V^{2 m^{2} g-1}$. 